\title{
Aminoterminal Propeptide of Type III Procollagen in Cord Blood and Amniotic Fluid of High-Risk Pregnancies: A Biochemical Approach to the Dynamic Assessment of Deviant Fetal Growth
}

\author{
PIET VANHAESEBROUCK, JOS KINT, HENDRIK VAN KETS, PAUL GOVAERT, \\ KOEN SMETS, PAUL DEFOORT, AND JULES LEROY
}

Department of Pediatrics and Neonatal Medicine [P.V., J.K., P.G., K.S., J.L.] and Department of Obstetrics and Gynecology [H.V.K., P.D.], University Hospital Gent, Gent, Belgium

\section{ABSTRACT}

\begin{abstract}
$\mathrm{N}$-terminal propeptide of type III procollagen (PIIINP) concentration was measured in cord serum, amniotic fluid, and maternal serum from high-risk pregnancies. The fetal PIIINP variability was shown to be independent of the maternal serum PIIINP values. Although a highly significant negative correlation was found between the fetal propeptide level and gestational age in both appropriate-for-gestationalage neonates $(n=504)$ and small-for-gestational-age infants $(n=98)$, the PIIINP concentration in cord serum or amniotic fluid of small-for-gestational-age infants was significantly lower compared with that of appropriate-for-gestational-age infants matched for postconceptional age. PIIINP assay may thus serve as a dynamic biochemical indicator of deviant fetal growth. The PIIINP results were also related to the severity or duration of intrauterine growth retardation, as indicated by significantly lower propeptide cord serum values in nonmalformed small-for-gestational-age infants with small head circumference, known as an index for the chronicity of fetal nutritional deprivation. Preeclampsia, maternal diabetes or smoking, and congenital anomalies appeared not to be associated with any alteration of fetal propeptide concentration, provided they did not cause fetal growth deceleration. The finding of extremely high cord serum PIIINP
\end{abstract}

IUGR rates among the most pressing problems in perinatology (1). To this end, there remains a need for objective measurement of fetal growth itself, independent of ultrasonographic in utero observation. During childhood, the serum concentration of PIIINP closely mirrors the shape of the growth velocity curve, but no significant correlation with either height or body weight

Received March 18, 1993; accepted February 1, 1994.

Correspondence and reprint requests: P. J. Vanhaesebrouck, M.D., Department of Pediatrics and Neonatal Medicine, University Hospital Gent, De Pintelaan, 185, B-9000 Gent, Belgium.

Supported in part by Grant 3.0020.91 from the Medical Research Foundation of Belgium (F.G.W.O.). values in six newborn infants with the Potter malformation sequence led to the speculation that large amounts of propeptides or their fragments usually are excreted by the fetal kidneys into the amniotic fluid. We suggest that determination of the PIIINP level in amniotic fluid or cord serum, obtained by amniocentesis and percutaneous umbilical sampling, may be a helpful adjunctive biochemical parameter in future research protocols assessing fetuses at risk for intrauterine growth retardation. (Pediatr Res 36: 71-76, 1994)

Abbreviations
PIIINP, N-terminal propeptide of type III
ABB, antibody-based RIA for PIIINP rec
predominantly the intact propeptide (Col
FAB, RIA for PIINP using Fab' antibody
are equally reactive with Col 1-3 and Col
SGA, small for gestational age
AGA, appropriate for gestational age
PI, ponderal index
OFC, occipitofrontal circumference
r, Spearman rank correlation coefficient
SD score, standard deviation score
IUGR, intrauterine growth retardation

was found in the normal childhood population (2-5). Our study on PIIINP in the late gestation fetus showed identical relations to fetal somatic growth rate of AGA infants during the second half of pregnancy (6). However, in the childhood studies, the PIIINP serum levels were signifcantly lower for age-matched patients with various growth disorders (e.g. growth hormone deficiency, Turner syndrome) (2-5). These observations prompted the hypothesis that assay of PIIINP concentration in cord serum or amniotic fluid of newborn infants may also be a method for quantitative assessment of deviant fetal growth in high-risk pregnancies and may allow us to determine the importance of the alleged phenomenon of 
uterine constraint and borderline nutrient supply as term approaches.

The aim of the present study was to assess the clinical usefulness of the PIIINP assay in cord serum and amniotic fluid for the detection of deviant fetal growth during the second half of high-risk pregnancies.

\section{METHODS}

Subjects. SGA infants form part of the computer database file of 602 neonatal records also used for the study of AGA infants (6). Ninety-eight of the 602 newborns were classified as SGA infants because their birth weight was below the 10th percentile (matched for sex and parity) according to the Dutch data on intrauterine growth (7). Among these SGA infants, $62.2 \%(n=61)$ were born after a pregnancy of less than 37 completed weeks. Cord serum was assayed in all SGA infants, and in 35 of them the PIIINP test could also be performed on paired amniotic fluid specimens.

Thirty-four newborns were the offspring of 29 (of 535) pregnancies complicated by preeclampsia, defined as systemic hypertension after the 20th wk of pregnancy (systolic or diastolic blood pressure $\geq 140$ or $90 \mathrm{~mm} \mathrm{Hg}$, respectively) with proteinuria $(>0.3 \mathrm{~g} / \mathrm{L})$, edema, or both. There were 12 single births from mothers with insulin-dependent diabetes, and 156 (29\%) of the 535 mothers were smokers during pregnancy, 46 of them "heavy" smokers (more than 20 cigarettes a day).

During the study, six infants with the Potter malformation sequence were studied for PIIINP cord serum levels. Three of them had bilateral renal cystic dysplasia, two had bilateral renal agenesis, and one had infantile polycystic kidney disease. All these infants died within $24 \mathrm{~h}$ because of fetal anuria and anhydramnios-related lung hypoplasia.

The PIIINP concentration was also determined in the serum of 58 mothers from our study group.

This study received the approval of the ethical committee at the University Hospital Gent. Informed consent was obtained from the parents before they participated in the study.

Sampling and storage procedures were identical with those described in our previous PIIINP study on healthy AGA infants (6).

Biochemical methods. Two different assay methods (regular PIIINP assay, i.e. RIAgnost P-III-P, and Fabmodified PIIINP assay; Behringwerke AG, Marburg, Germany) were used for the quantification of the PIIINP concentrations in cord serum and amniotic fluid. The crossover interrelationship between FAB and $\mathrm{ABB}$ assays for PIIINP concentrations in both fetal biologic fluids was studied on the whole cohort of infants, and their interchangeability for clinical research on fetal growth rate was demonstrated in our former report (6). Therefore, only the largest number of identical test results available, FAB assay of cord serum and ABB assay of amniotic fluid, will be considered in the further analysis of high-risk pregnancies.

Statistical methods. Correlations between PIIINP levels and auxanologic data were evaluated by Spearman rank correlation analysis (8). A multivariate regression analysis was used to define those variables exerting influence independent of others. The Mann-Whitney $U$ test was used for comparing prognostic groups.

\section{RESULTS}

Cord serum PIIINP levels (FAB assay). Mere visual inspection of a simple $x-y$ plot of PIIINP serum level $(y)$ on birth weight $(x)$ shows an obvious difference of the relation between the serum PIIINP level of SGA infants $(n=98)$ and birth weight compared with that for AGA infants $(n=504)$ (Fig. 1). Upon comparison of the data in AGA infants with those in SGA infants matched for fetal lifetime, highly significant differences $(p<0.0001)$ in serum PIIINP were found, although even in the SGA group a significant negative correlation $(p<0.0001)$ between PIIINP concentration and gestational age remained (covariance analysis) (Table 1).

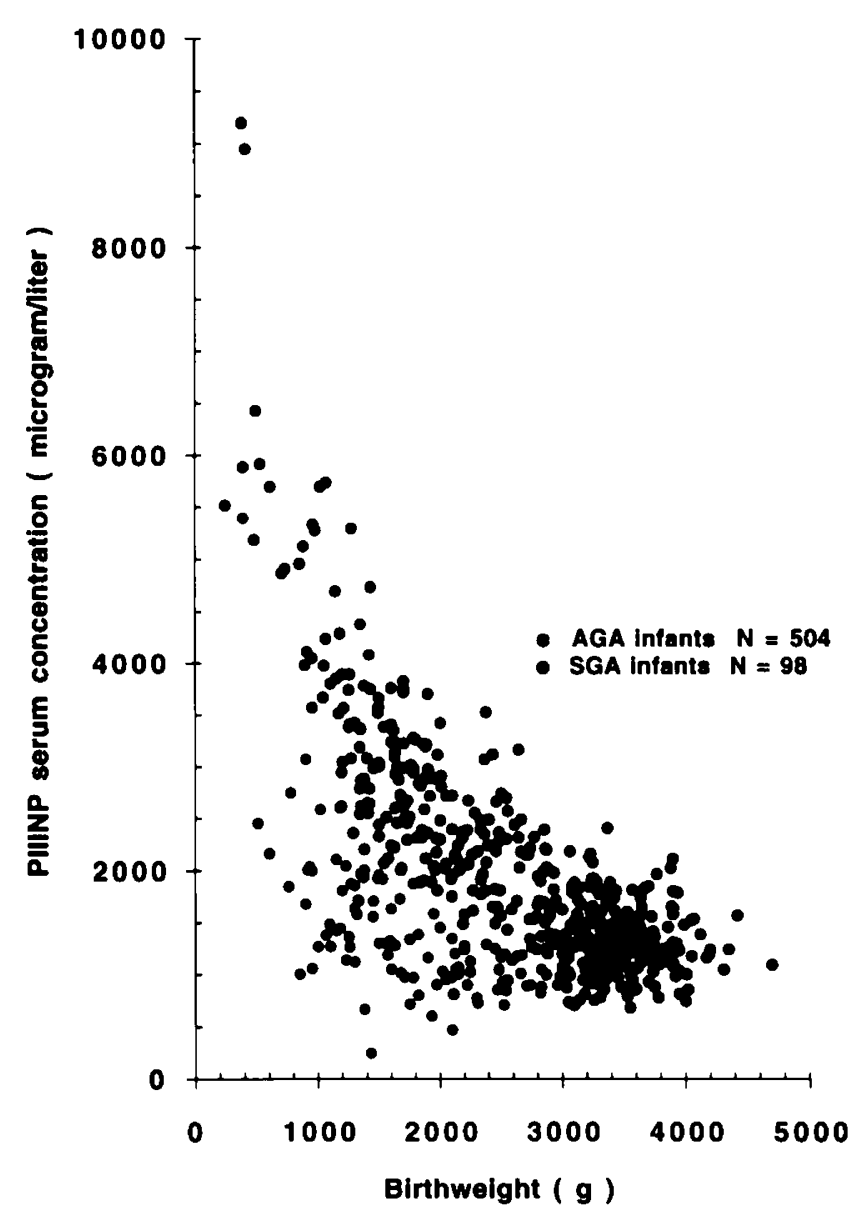

Figure 1. Relation of cord serum FAB assay PIIINP level (y) to birth weight (x) in 602 neonates. Gray circles, AGA infants; black circles, SGA infants. 
Table 1. Comparison of FAB assay PIIINP cord serum levels in $A G A$ and $S G A$ infants matched for postconceptional lifetime

\begin{tabular}{lccc}
\hline Gestational age group & AGA infants* & SGA infants* & $p$ value $\dagger$ \\
\hline 38-41 wk & & & \\
Number of infants & 230 & 30 & \\
Gestational age (wk) & $39.0 \pm 0.8$ & $38.8 \pm 0.7$ & NS \\
Birth weight (g) & $3419 \pm 345$ & $2448 \pm 313$ & $<0.0001$ \\
PIIINP ( $\mu \mathrm{g} / \mathrm{L}$ ) & $1280 \pm 202$ & $955 \pm 179$ & $<0.0001$ \\
33-36 wk & & & \\
Number of infants & 104 & 40 & \\
Gestational age (wk) & $34.5 \pm 1.1$ & $34.4 \pm 1.2$ & NS \\
Birth weight (g) & $2379 \pm 457$ & $1571 \pm 307$ & $<0.0001$ \\
PIIINP ( $\mu$ /L) & $2153 \pm 323$ & $1374 \pm 383$ & $<0.0001$ \\
29-32 wk & & & \\
Number of infants & 95 & 15 & \\
Gestational age (wk) & $30.8 \pm 1.0$ & $30.6 \pm 0.9$ & NS \\
Birth weight (g) & $1604 \pm 297$ & $1085 \pm 172$ & $<0.0001$ \\
PIINP ( $\mu \mathrm{g} / \mathrm{L})$ & $3044 \pm 431$ & $1760 \pm 441$ & $<0.0001$ \\
\hline
\end{tabular}

*Values are mean $\pm \mathrm{SD}$.

$\dagger$ Mann-Whitney $U$ test.

PI (birth weight/birth length $\left.{ }^{3} \times 100\right)$ is frequently used for rough differentiation of chronic (or symmetric) and subacute (or asymmetric) IUGR $(1,9,10)$. When covariation with gestational age was taken into account, the significant $(p<0.0001)$ correlation coefficient between PIIINP level and PI in AGA as well as in SGA infants was no longer statistically meaningful, even after exclusion of the infants with structural anomalies.

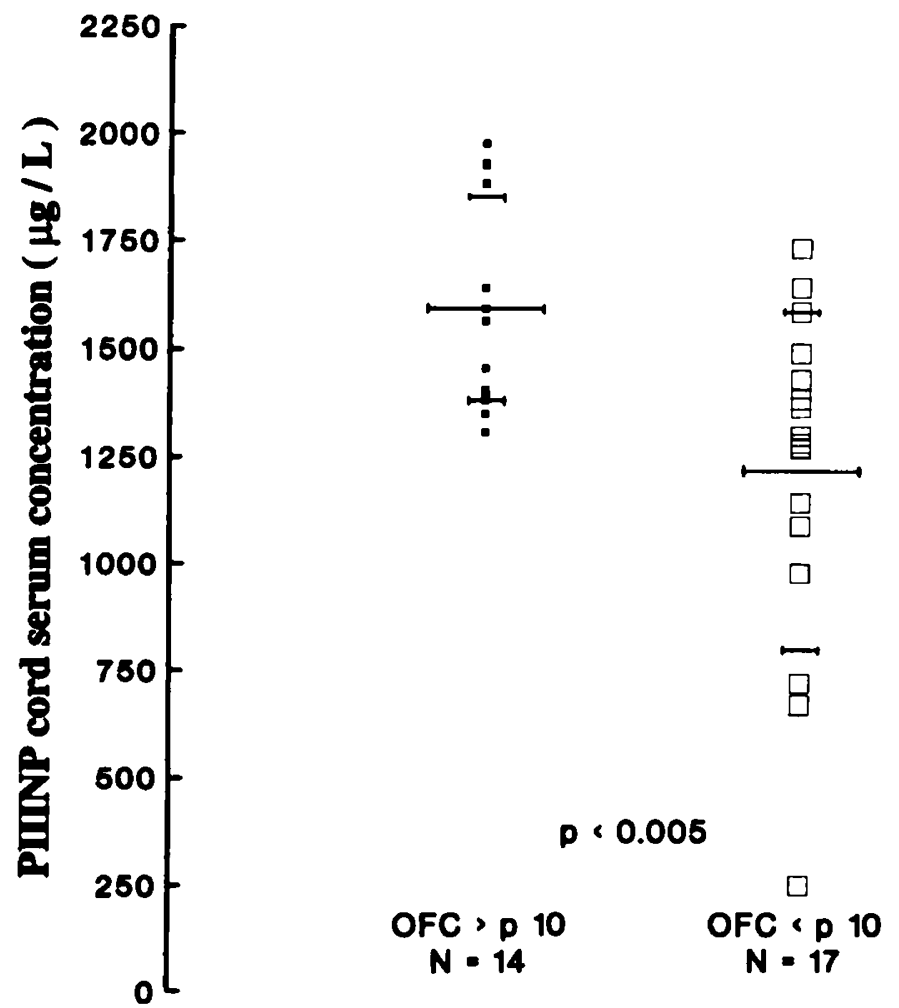

Figure 2. PIIINP cord serum levels in two groups of age-matched nonmalformed SGA infants: left, OFC $>$ the 10th percentile $(p 10)(n=$ 14); right, OFC $<p 10)(n=17)$. Mean and SD are indicated by horizontal lines.
Two groups of age-matched SGA infants (mean gestational age: $34.1 \pm 1.46$ versus $34.2 \pm 1.25 \mathrm{wk} ; p=0.85$ ) without congenital anomalies were compared with respect to their PIIINP serum levels (Fig. 2). The first group had an OFC above the 10th percentile [Lubchenco's standards (11)]; the second one had an OFC below the 10th percentile. The PIIINP serum level in the second group was significantly $(p<0.005)$ lower than that in the first group with unaltered brain volume (mean \pm SD PIIINP level: $1619 \pm 249$ versus $1213 \pm 380 \mu \mathrm{g} / \mathrm{L}$ ).

Comparison of age-matched SGA infants with $(n=20)$ and without $(n=70)$ congenital malformations revealed no significant difference in propeptide value $(p=0.33)$. There was also no statistical difference $(p=0.12)$ between the PIIINP levels in two groups of SGA infants matched for gestational age but differing only by single ( $n$ $=47)$ or multiple birth $(n=24)$ circumstances. The individual PIIINP serum levels (FAB assay) of all SGA infants $(n=98)$ are plotted on the "standard" (6) percentile chart (Fig. 3).

Thirty $(88 \%)$ of 34 offspring of preeclamptic mothers were born prematurely. Twenty-two of the 34 newborns were SGA. Covariance analysis of PIIINP values with gestational age revealed that only IUGR and not preeclampsia was related to the PIIINP variability $(p=0.94, F$ ratio $=0.007$ ).

None of the newborns $(n=12)$ from mothers with diabetes were growth retarded and none had congenital anomalies. Their mean PIIINP serum level was not sig-

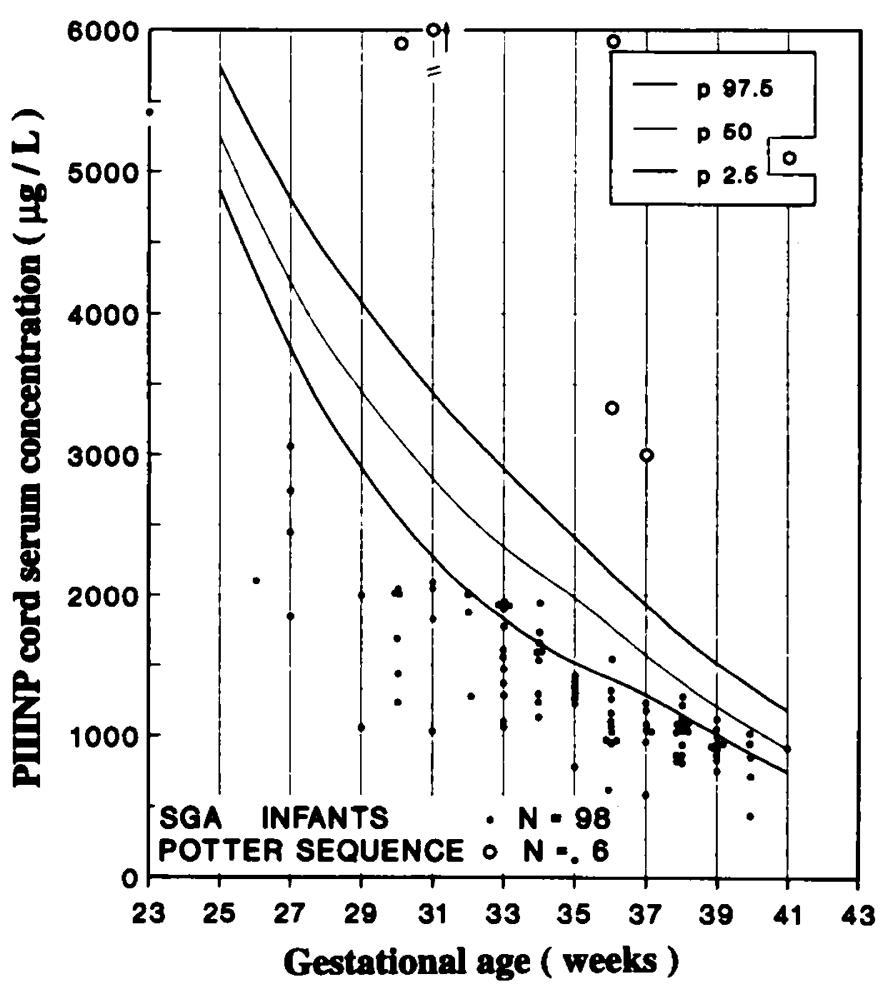

Figure 3. Percentile chart for cord serum FAB assay PIIINP levels of AGA infants in relation to gestational age. Serum PIIINP values of 98 SGA infants $(\cdot)$ and six infants with Potter sequence of malformation $(O)$ are plotted individually. 
nificantly $(p=0.66)$ different from the level in agematched (33-40 wk) AGA infants $(n=336)$.

The cord serum PIIINP level in relation to smoking revealed no significant differences between infants of nonsmokers, moderate smokers, and heavy smokers matched for gestational age (Kruskall-Wallis analysis). However, PIIINP value in the serum of infants of heavysmoking mothers was significantly $(p<0.001)$ lower in their SGA offspring compared with that in age-matched AGA infants.

Although two of the six neonates with the Potter malformation sequence were also growth retarded, the PIIINP cord serum level in all was extremely high and well outside the normal range for the respective gestational ages (Fig. 3). Three of the six patients with Potter sequence belonged to multiple birth pregnancies. The PIIINP level in cord blood of the second healthy twin was within the normal range for gestational age.

Amniotic fluid PIIINP levels (ABB assay). A simple $\mathrm{x}-\mathrm{y}$ plot of amniotic fluid PIIINP level $(\mathbf{y})$ on birth weight $(\mathbf{x})$ illustrates that the amniotic fluid propeptide level probed by the ABB assay in SGA infants $(n=35)$ is apparently different from that in AGA infants $(n=268)$ (Fig. 4).

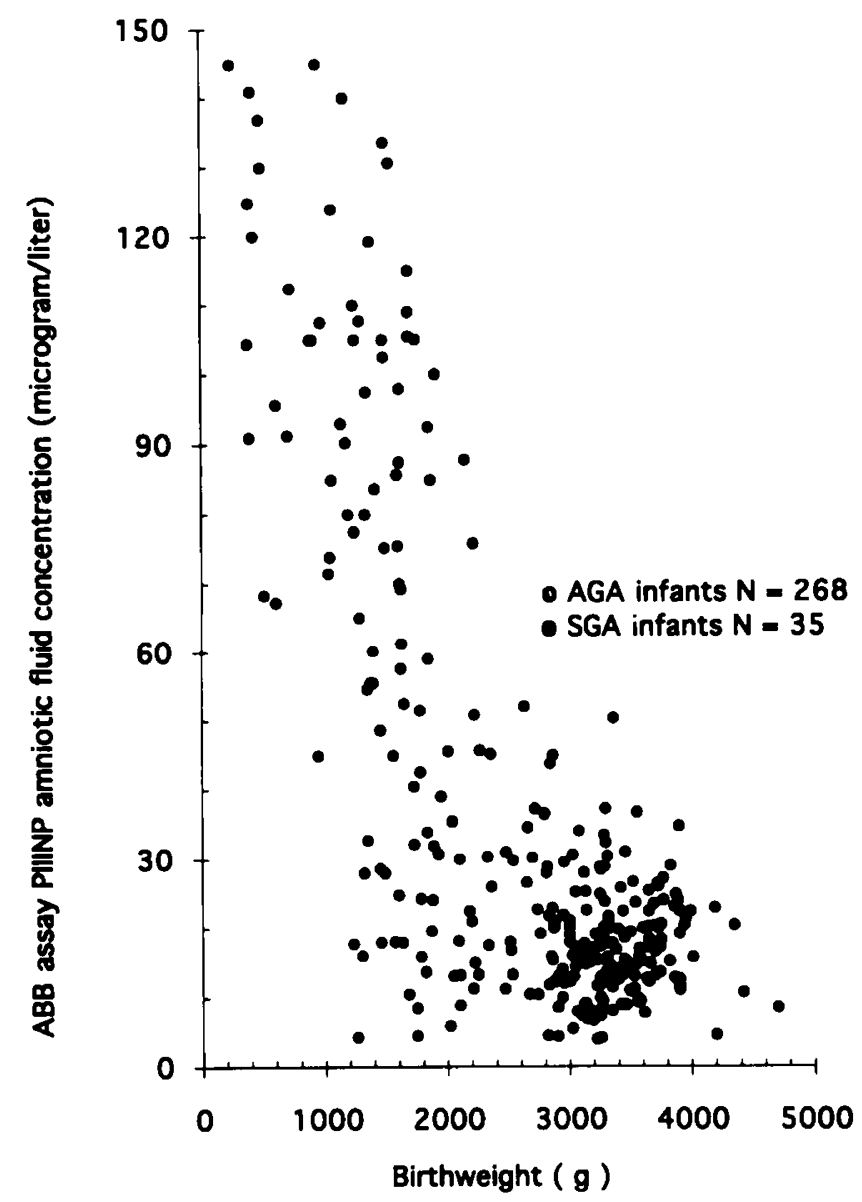

Figure 4. Relation of amniotic fluid ABB assay PIIINP level (y) to birth weight (x) in 303 neonates. Gray circles, AGA infants; black circles, SGA infants.
When the data of SGA infants were compared with those in AGA infants matched for postconceptional lifetime, significant differences in amniotic fluid PIIINP level were found for full-term (37-40 wk: $p<0.05)$ as well as for preterm infants (33-36 wk: $p<0.0001)$. However, covariance analysis for amniotic fluid PIIINP concentration with gestational age was still significant in SGA infants $(p<0.001)$.

The relationship of the amniotic fluid PIIINP level to birth weight, expressed as SD score, showed no significant correlation for the total group of infants $(n=303$; $\left.r_{\mathrm{s}}=-0.0002, p=0.99\right)$. A significant, albeit weak, correlation with the SD score was found for PIIINP level of neonates with an SD score less than $-2(n=25$; $\mathrm{r}_{\mathrm{s}}=0.41, p<0.05$ ). The PI of age-matched SGA infants did not correlate with the amniotic fluid propeptide value.

The individual ABB assay PIIINP levels in amniotic fluid of all SGA infants $(n=35)$ are plotted on the "standard" (6) percentile chart (Fig. 5).

Maternal serum PIIINP levels (FAB assay). PIIINP serum levels (FAB assay) were measured in 58 maternal blood specimens collected during labor or immediately after delivery. The serum PIIINP concentrations from mothers $(n=28)$ of uncomplicated, single, term pregnancies were significantly higher (mean \pm SD: $127.3 \pm$ $8.1 \mu \mathrm{g} / \mathrm{L}$ ) than known values (12) in nonpregnant adults (20-65 y old: $39 \pm 12 \mu \mathrm{g} / \mathrm{L}$ ). There was a significant $(p<0.001)$ steep rise in PIIINP concentration near term in comparison with the level in mothers giv-

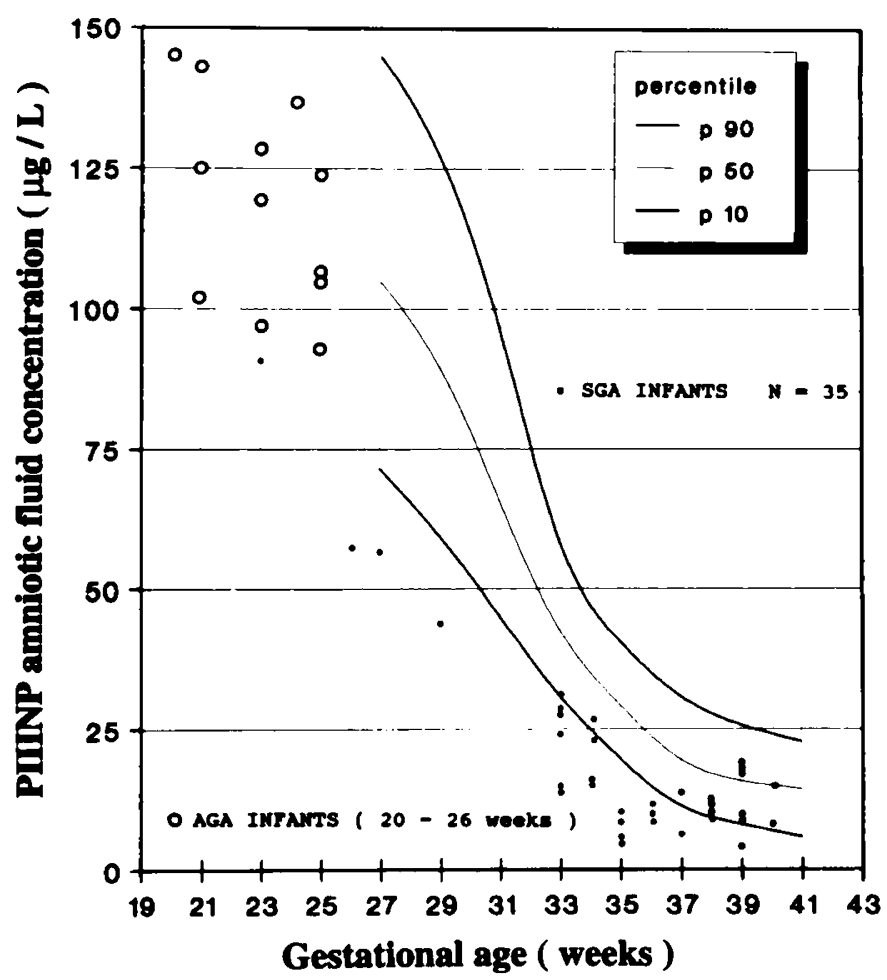

Figure 5. Percentile chart for amniotic fluid ABB assay PIIINP levels of AGA infants in relation to gestational age. The values for 20- to 26-wk AGA fetuses $(O)$ and for SGA infants $(\bullet)$ are plotted individually. 
ing birth between the 25th and 37th wk of pregnancy (mean \pm SD: $71.2 \pm 6.1 \mu \mathrm{g} / \mathrm{L}$ ). PIIINP levels in the serum of either mothers with twin pregnancies $(n=10)$ or mothers suffering from preeclampsia $(n=3)$ (mean \pm SD: $157.9 \pm 14.2 \mu \mathrm{g} / \mathrm{L})$ were significantly higher $(p<$ $0.001)$ than the ones in mothers with age-matched subsequent uncomplicated singleton preterm-birth pregnancies.

\section{DISCUSSION}

In our previous study (6), it was shown that PIIINP level in cord serum and amniotic fluid of AGA fetuses is directly correlated with age-related fetal somatic growth rate during the second half of pregnancy. The results presented here show in addition that cord serum or amniotic fluid PIIINP concentration assay enables the investigator to discern deviant from normal fetal growth, inasmuch as SGA fetuses have significantly lower propeptide values in comparison with age-matched AGA infants. This is of paramount importance, because this biochemical approach to the evaluation of fetal growth may have the singular advantage of discovering growth rate decelerations at one point in time and predicting a probable outcome, whereas conventional auxologic methods such as ultrasound anthropometry can only reveal the point in physical growth already reached at the time of observation. Calculation from such anthropometric growth rates are necessarily retrospective in nature (13). To what extent propeptide levels in cord serum or amniotic fluid may be prompt measures of a change in fetal growth activity, well before clinical or ultrasonographic changes become apparent, must still be investigated by more detailed prospective studies of individual pregnancies at high risk for subsequent IUGR. The study of the correlation of PIIINP levels with Doppler flow resistance indices of fetal cerebral vessels-shown to reach adequate accuracy in predicting IUGR in high-risk fetuses $(14,15)$-deserves priority, inasmuch as it may be of great value in the early identification of IUGR before irreversible fetal brain damage has occurred.

Our results are at variance with the findings reported by Yunoki et al. (16), who found no difference of PIIINP quantities in cord serum between AGA and SGA neonates matched for postconceptional age. This inconsistency may be explained by the fact that in the Yunoki study interferences by either maternal or fetal auxanologic data or complications of pregnancy and labor were not taken into account, and SGA infants with fetal malformations or those born to hypertensive mothers were excluded. Moreover, the majority of the SGA infants $(n$ $=22$ ) in that study were near- or full-term neonates. At the end of pregnancy, as demonstrated by the results presented (Fig. 3) and as also assumed by Yunoki et al. (16), systemic collagen synthesis must stabilize in SGA as well as in AGA fetuses.

The degree of IUGR cannot be quantitatively assessed relating PI to serum propeptide value. This finding is in agreement with the finding that at any gestational age PIIINP levels are not correlated with body length. Similar conclusions were reached in studies on propeptide serum level and linear growth velocity in later childhood (2-5). Significantly lower propeptide values detected in nonmalformed SGA infants with a small OFC compared with age-matched SGA infants with normal head circumference suggest that the results of the PIIINP assay are related to the severity and duration of declining fetal growth. Interestingly, the multivariate analysis presented in our previous report on PIIINP assay (6) shows that, in addition to gestational age, head circumference has a significant independent positive correlation with the fetal PIIINP serum concentration. Small OFC in nonmalformed SGA infants-a well-recognized index of " chronic" nutritional deprivation-is thought to be induced, during longstanding uteroplacental insufficiency, by the collapse of the preferential brain perfusion (1). The latter hypothesis has received valuable support from the results of in vivo studies of Doppler flow in the internal carotid artery of growth-retarded human fetuses (14).

Not preeclampsia itself but rather the ensuing fetal growth deceleration is solely responsible for the decrease in fetal cord serum propeptide levels. It is hardly surprising that AGA infants of diabetic mothers have propeptide values similar to age-matched controls, because their often higher PI are mainly due to fat accumulation instead of higher accretion of protein (17). That serum PIIINP concentrations are independent of serum levels of IGF-I is in agreement with such a proposition (16).

The fact that maternal serum PIIINP values rise toward the end of a normal pregnancy and are still higher in a complicated pregnancy (e.g. multiple birth, preeclampsia) helps repudiate the assumption that transplacental transfer of propeptides would explain the higher PIIINP levels in premature infants (present data; 18).

Congenital anomalies seemed not to be associated with any alteration of propeptide concentration, provided they did not cause intrinsic fetal growth deceleration. However, the finding of extremely high cord serum levels of PIIINP in six newborn infants with various types of the Potter sequence of malformation prompts a separate discussion. It may be surmized that PIIINP accumulation occurs in the fetal circulation due to the absence of renal function and that normally large amounts of procollagen propeptides or their fragments may be excreted by the fetal kidneys into the amniotic fluid. However, Keller $e t$ al. (19) have demonstrated that, although serum PIIINP values (ABB and FAB assay) of adult kidney patients correlated directly $\left(r_{\mathrm{s}}=0.41, p=0.001\right)$ with serum creatinine levels and were significantly higher than in the serum of healthy volunteers, PIIINP elimination did not depend on renal function. These authors assumed that increased intrarenal collagen production due to progressive renal fibrosis could explain the elevated PIIINP serum levels in all kidney patients. This hypothesis is obviously not applicable to our neonates with bilateral renal agenesis. The speculation that propeptides may be 
excreted by the normal fetus into the amniotic fluid prompted the study of the PIIINP concentration in that complex body fluid. Kinetic study of release metabolism and uptake of the propeptides from amniotic fluid is needed to prove this point, although rather strong evidence is provided by the proportionality of the PIIINP concentrations in paired samples of cord serum and amniotic fluid (6). Chromatographic methods should also be used to detect the unknown distribution of the different antigenic forms of PIIINP in biologic fluids of newborn infants (18). The PIIINP peptides in amniotic fluid may also at least in part originate from other fetal body fluids or tissues such as lung fluid or amniochorial cells in the fetal membranes.

It can be concluded that fetal $\mathrm{N}$-terminal propeptide levels of procollagen type III in cord serum or in amniotic fluid not only reflect the fetal somatic growth potential in AGA infants, but may also be a dynamic biochemical indicator of decelerating fetal growth and an index of the severity of IUGR. Because PIIINP, to identify the SGA fetus, is discriminatory at the statistically defined cutoff of the 10th percentile of the fetal weight chart, this arbitrarily chosen limit seems to be a physiologically defensible selection criterion. Therefore, we suggest that determination of the PIIINP level in amniotic fluid or cord serum obtained by amniocentesis and percutaneous umbilical sampling may be a helpful adjunctive biochemical index in prenatal research on fetuses at risk for IUGR (20). It may possibly also help to monitor therapeutic interventions under study such as maternal oxygen therapy, hyperalimentation, or timely termination of pregnancy in prenatally detected fetal growth deceleration in fetuses at risk for irreversible fetal brain damage or intrauterine death (21-24).

Acknowledgments. The authors thank the nursing staff of the obstetrical and neonatal intensive care departments of the University Hospital and "Bijloke" Hospital Gent for their help in so efficiently organizing the collection of samples. The skillful technical support of Adil Huys and Odette Coessens is greatly appreciated. Adil Huys died prematurely during the last months that this work was carried out. This paper is dedicated to his commemoration. We also thank Georges Van Maele for invaluable advice and assistance with statistical data analysis.

\section{REFERENCES}

1. Prader A 1983 Croissance du foetus et de l'enfant. Arch Fr Pediatr 40:365-373

2. Danne T, Gruters A, Schuppan D, Quantas N, Enders I, Weber B 1989 Relationship of procollagen type III propeptide-related antigens in serum to somatic growth in healthy children and patients with growth disorders. J Pediatr 114:257-260

3. Vainionpaa L, Risteli L, Lanning M, Myllyla V, Risteli J 1991 Aminoterminal propeptide of type III procollagen in cerebrospinal fluid. Variation with age and in childhood leukemia. Clin Chim Acta 203:47-56

4. Danne T, Gruters A, Schnabel K, Burger W, L'Allemand D, Enders I, Helge $\mathrm{H}$, Weber 1988 Long-term monitoring of treatment with recombinant human growth hormone by serial determinations of type III procollagen-related antigens in serum. Pediatr Res 23:167-171

5. Trivedi P, Hindmarsh P, Risteli J, Risteli L, Mowat AP, Brook LG 1989 Growth velocity, growth hormone therapy, and serum concentrations of the amino-terminal propeptide of type III procollagen. J Pediatr 114:225-230

6. Vanhaesebrouck P, Kint J, Dhont M, de Praeter C, Leroy JG 1994 Aminoterminal propeptide of type III procollagen in cord blood and amniotic fluid of appropriate-for-gestational-age infants: a predictor of age-related fetal growth rate. Pediatr Res 36:64-70

7. Kloosterman GJ 1969 Over intra-uteriene groei en de intra-uteriene groeicurve. Maandschr Kindergeneesk 37:209-225

8. Glantz SA 1981 Primer of Biostatistics. McGraw-Hill, New York, pp 217-221

9. Walther FJ, Ramaekers LHJ 1982 Neonatal morbidity of SGA infants in relation to their nutritional status at birth. Acta Paediatr Scand 71:437-440

10. Wolfe HM, Brans YW, Gross TL, Bhatia RK, Sokol RJ 1990 Correlation of commonly used measures of intrauterine growth with estimated neonatal body fat. Biol Neonate 57:167-171

11. Lubchenco LO, Hansman C, Boyd E 1966 Intrauterine growth in length and head circumference as estimated from live births at gestational ages from 26 to 42 weeks. Pediatrics $37: 403-408$

12. Rohde H, Vargas L, Hahn E, Kalbfleisch H, Bruguera M, Timpl R 1979 Radioimmunoassay for type III procollagen peptide and its application to human liver disease. Eur J Clin Invest 9:451-459

13. Milner RD, Hill DJ 1989 Fetal growth signals. Arch Dis Child 64:53-57

14. Wladimiroff JW, Tonge HM, Stewart PA 1986 Doppler ultrasound assessment of cerebral blood flow in the human fetus. Br J Obstet Gynaecol 93:471-476

15. Kurjak A, Alfirevic Z, Rizzo G, Arduini D 1989 Uteroplacental and fetal circulation in intrauterine growth retardation. In: Kurjak A, Beazley JM (eds) Fetal Growth Retardation: Diagnosis and Treatment. CRC Press, Boca Raton, FL, pp 119-146

16. Yunoki H, Yagi H, Nagashima K, Noji T, Miyake H, Kuroume T 1990 $\mathrm{N}$-terminal propeptide of type III procollagen concentrations in the cord blood: an index of newborn maturity. Biol Neonate 58:264-270

17. Widdowsown EM 1974 Changes in body proportion and composition during growth. In: Davis JA, Dobbing J (eds) Scientific Foundations of Paediatrics. WB Saunders, Philadelphia, pp $44-45$

18. Risteli L, Puistola U, Hohtari H, Kauppila A, Risteli J 1987 Collagen metabolism in normal and complicated pregnancy: changes in the aminoterminal propeptide of type III procollagen in serum. Eur J Clin Invest 17:81-86

19. Keller F, Rehbein C, Schwarz A, Fleck M, Hayasaka A, Schuppan D, Offermann G, Hahn EG 1988 Increased procollagen III production in patients with kidney disease. Nephron 50:332-337

20. Okamura K, Endoh H, Watanabe T, Tanigawara S, Iwamoto M, Murotsuki J, Yajima A 1989 Reevaluation of nonstress test by umbilical venous blood profile using cordocentesis. Fetal Ther 4:146-151

21. Nicolaides KH, Campbell S, Bradley RJ, Bilardo C, Soothill PW, Gibb D 1987 Maternal oxygen therapy for intrauterine growth retardation. Lancet 1:942-944

22. Johnson MP, Evans MI 1987 Intrauterine growth retardation: pathophysiology and possibilities for intrauterine treatment. Fetal Ther 2:109-122

23. Spatling L, Spatling G 1988 Magnesium supplementation in pregnancy: a double-blind study. Br J Obstet Gynaecol 95:120-125

24. Lejeune C, Thizon-de Gaulle I, Retbi JM 1992 Problèmes cliniques posés par le nouveau-né atteint de RCIU. In: Journées Parisiennes de Pédiatrie. Flammarion Médecine-Sciences, Paris, pp 49-56 